\title{
A test-proven As-grown-Generation (A-G) model for predicting NBTI under use-bias
}

\author{
Z. Ji (z.ji@ljmu.ac.uk), J. F. Zhang, L. Lin, M. Duan, W. Zhang, X. Zhang, R. Gao, B. Kaczer ${ }^{(1)}$, J. Franco ${ }^{(1)}$, T. Schram $^{(1)}$, \\ N. Horiguchi ${ }^{(1)}$, S. De Gendt ${ }^{(1)}$, and G. Groeseneken ${ }^{(1)}$ \\ School of Engineering, Liverpool John Moores University, Liverpool L3 3AF, UK. ${ }^{(1)}$ IMEC, Leuven B3001, Belgium
}

Introduction: The gap between modelling and real performance has been identified as a major constraint for design optimisation [1] and the inaccuracy of NBTI models contributes to it. When developing a NBTI model, short-term accelerated tests are usually used to extract model parameters and it is a common practice to 'qualify' a model by showing it fits well with test data. The models 'qualified' in this way, such as the reaction-diffusion (R-D) framework [2], cannot predict the long-term NBTI under low use-bias for both SiON and HKMG processes (Figs.1a\&b). There is a pressing need for delivering the original mission: reliably predicting long term NBTI at low use-bias, based on a model extracted from Vg-accelerated short tests.

Key advance of this work: The As-grown-Generation (A-G) model [3] has successfully demonstrated its excellent predicting capability on device reliability and variability under DC NBTI [4] and HCI conditions [5]. This work demonstrates, for the first time, (i) both $D C$ and $A C N B T I$ under use-overdrive Vg_ov can be reliably predicted from the A-G model extracted from Vg-accelerated short tests (Figs. 1cd), and (ii) the same model can also predict the NBTI under variable operational workload (Fig.2), needed for dynamic voltage scaling power management [6]. The model needs only three fitting parameters. We emphasize that the A-G model is extracted from the accelerated short DC tests and the test data at low biases in lower panels of Fig.1c\&d were not used for fitting. This success is achieved after a detailed understanding of different types of defects and their contributions to NBTI, based on direct measurements of each type of defects (Table I), as described below.

Measuring different types of defects: Devices from three different processes are used, including HKMG and SiON (Table II). Measurement with $3 \mu \mathrm{s}$ speed is used for both DC\&AC NBTI under $125{ }^{\circ} \mathrm{C}$. Early works [3] reported that as-grown hole trap (AHT) and generated defects (GD) are located below and above the energy level $\mathrm{E}(\mathrm{VgGD})$ (Fig.3) respectively, allowing their separation. For unipolar AC stress, the defects above $\mathrm{E}(\mathrm{Vg}=0 \mathrm{~V})$ do not discharge once generated, but those between $\mathrm{E}(\mathrm{VgGD})$ and $\mathrm{E}(\mathrm{Vg}=0)$ do. As a result, the GDs are further separated into two parts: the anti-neutralization positive charges (ANPC) above $\mathrm{E}(\mathrm{Vg}=0 \mathrm{~V})$ and the cyclic positive charges (CPC) between $\mathrm{E}(\mathrm{VgGD})$ and $\mathrm{E}(\mathrm{Vg}=0 \mathrm{~V})$ (Fig.3).

The Vg waveform for their measurement under DC and AC stress is given in Figs.4a\&d, respectively. ' 2 ' in Fig.4b is the sum of all defects. By biasing at VgGD (Fig. 3), all AHTs are discharged, so that AHT (' $\Delta$ ' in Fig.4c) $=$ '资' -' $x$ '. The device was then biased at $\mathrm{Vg}=0 \mathrm{~V}$ to discharge $\mathrm{CPC}$, so that $\mathrm{CPC}\left({ }^{\prime} \triangleleft\right.$ ' in Fig.4c) $={ }^{\prime} \mathrm{x}^{\prime}-{ }^{\prime} \diamond$ ' and ANPC=' $\diamond$ ' (Figs.4c). For AC stress, total $\Delta V$ th are measured on two edges: one from zero to Vgst ('o') as "End-of-Recovery (EoR)" and the other from the opposite edge (' $\square$ ') as "End-of-Stress (EoS)" (Figs.4d\&e). This gives two sets of AHTs in Fig.4f: AHT_EoS $\left({ }^{\prime} \Delta^{\prime}\right)={ }^{\prime} \square^{\prime}-{ }^{\prime} x^{\prime}$ and AHT_EoR( $\left(\nabla^{\prime}\right)={ }^{\prime} \mathbf{0} \mathbf{-}^{\prime} \mathrm{x}^{\prime}$ '. The CPC and ANPC were evaluated in the same way as that after DC stress. Their properties and contributions to NBTI are examined next.

Anti-neutralization positive charges (ANPC): For the same effective stress time, the same ANPC was obtained for DC and AC stresses at different frequencies (Fig.5a), as ANPC does not neutralize during AC stress. ANPC generation follows power law against both stress time (Fig.5b) and stress Vgst_ov (Fig.5c) and the exponents are independent of $\mathrm{Vg}$ and stress time, laying the foundation for reliable prediction. ANPC are modelled with three fitting parameters: $\mathrm{g}_{0}, \mathrm{~m}, \mathrm{n}$ (eq.1, Table I.)

As-grown hole traps (AHT): Under DC stress, more AHTs are charged at higher $|\mathrm{Vg}|$ (Figs.6a\&b), but the normalized kinetics is the same (Fig.6c). Under AC stress, AHT EoS reduces for higher frequency (Fig.7a), because of shorter charging time, tch=period/2. With the same tch, AHT_EoS agrees well with AHT_DC (Fig.7c). Charging AHTs can be fully modelled by the kinetics (Fig.6c) with its saturation level taken from Fig.6b. The efficient discharging under Vg leads to AHT_EoR $\approx 0$ for all frequencies (Fig.7b). This explains AHT_EoS=AHT_DC for the same tch, since charging restarts from $\sim$ zero in each cycle. The discharge kinetics is independent of $\mid$ Vgst ov $\mid$ (Fig.8a\&b) and used for modelling discharge.
Cyclic positive charges (CPC): $\mathrm{CPC}$ is the same for $\mathrm{DC}$ and $\mathrm{AC}$ initially $(<50 \mathrm{sec}$, Fig.9a), but CPC_DC saturates at a higher level eventually. To understand this, $\mathrm{CPC}_{-} \overline{\mathrm{D} C}$ was neutralized and recharged (Fig.9b). CPC can be filled to saturation much faster in the recharging compared with $1^{\text {st }}$ DC stress, confirming they are generated defects. Moreover, these generated CPC clearly has two components: i) fastcharging CPC (fCPC) recharged fully within $1 \mu \mathrm{s}$, and ii) slow-charging CPC (sCPC) only starts recharging after $5 \mathrm{~ms}$ and reach saturation after $10 \mathrm{~s}$. Their different dependence on stress Vg in Fig.10a supports that they are different defects. For DC NBTI, sCPC charging is modelled by the kinetics in Fig.9b. For AC NBTI, sCPC contributes little to charging (Fig.9a) as total $\mathrm{CPC} \mathrm{AC}=\mathrm{fCPC}$, because discharging is far more efficient than charging (Fig.10b V.S. Fig.9b). There is no need to model sCPC for AC NBTI, therefore. In contrast, charging fCPC is far more efficient than discharging (Fig.9b V.S. Fig.10c) and contributes to AC NBTI. Similar to AHTs, fCPC is modelled by the saturation level in Fig.10a and the discharging kinetics in Fig.10c.

Aging Prediction: Four Vg-accelerated short (1ks) DC stresses (Fig.1c) were carried out to extract the A-G model (Table I), giving 3 fitted parameters in Table II. The model can successfully predict both DC and AC NBTI under use- $\mathrm{Vg}$ ov (Figs.1c\&d), delivering the original mission of NBTI modelling. The NBTI under variable operation Vg_ov is also successfully predicted (Fig.2). Moreover, the A-G model predicts the frequency (Freq) and duty-factor (DF) dependence under operation condition well (Figs.11a\&b). The contributions of different defects are also shown in Figs.11a\&b. AHT is mainly responsible for the Freq- and DF-dependence of $\Delta V$ th_EoS. A higher Freq or smaller DF reduces the charge time at 'End-of-Stress' and in turn fills less AHTs (Fig.7sa\&b). At 'End-of-Recovery', however, AHTs $\approx 0$ for all frequencies due to efficient discharging (Fig.7b), resulting in the well-known frequency-independence of NBTI [7]. Over $1 \mathrm{MHz}, \mathrm{AHT} \approx 0$ and $\Delta \mathrm{Vth} \_\mathrm{EoS}=\Delta \mathrm{Vth} \_$EoR. Both $\mathrm{ANPC}$ and $\mathrm{fCPC}$ are frequency independent.

Process independence: The A-G model was applied to two other processes to prove it is not process specific. The measured Freq- and DFcharacteristics under low Vg ov agree well again (Figs.12a-d) with the predicted ones using A-G model extracted from the short DC stresses at high biases, in Table II.

An analysis of the success: To understand why A-G model can predict and early models [2] cannot, it is realized that the charging or generation of some defects will saturate with time during aging, like AHTs (Fig.6c) and CPCs (Figs.9b). However, ANPC does not saturate (Fig.5) and thus controls the long term aging. The A-G model's success comes from its accurate separation of ANPC from the rest of defects, enabling the reliable prediction from accelerated $\mathrm{Vg}$ to use $\mathrm{Vg}$ ov. Only one non-saturating aging kinetic is needed: a power law with $\mathrm{Vg}$ - and time-independent exponents for ANPC (Fig.5). The R-D framework [2] has to use two separate non-saturating kinetics with more fitting parameters, because the real non-saturating component was not properly separated out. The contamination of non-saturating defects by the saturated ones results in erroneous power exponents and prediction.

Conclusions: For the first time, we demonstrate that A-G model extracted from short Vg-accelerated stresses can predict both long term DC and AC NBTI under low and dynamic operation Vg. This is achieved by successfully separating non-saturating defects from the saturating ones, allowing reliable extraction of power exponents needed for long term prediction. Unlike R-D model, A-G model does not require solving differential equations for AC NBTI. This saves computation time significantly, especially for high-frequency that needs small time-step, and makes it readily implementable in SPICE-like simulators.

Acknowledgement: This work is supported by EPSRC of UK (EP/L010607/1). [1] G. Yeap, IEDM, 2013. [2] S. Desai, et al, IRPS 2013. [3] Z. Ji, et al, IEDM, 2013. [4] M. Duan, et al, VLSI, 2014. [5] M. Duan, et al, submitted to VLSI, 2015. [6] P. Mercati et al, DAC, 2013. [7] R. Fernandez, et al, IEDM, 2006. 

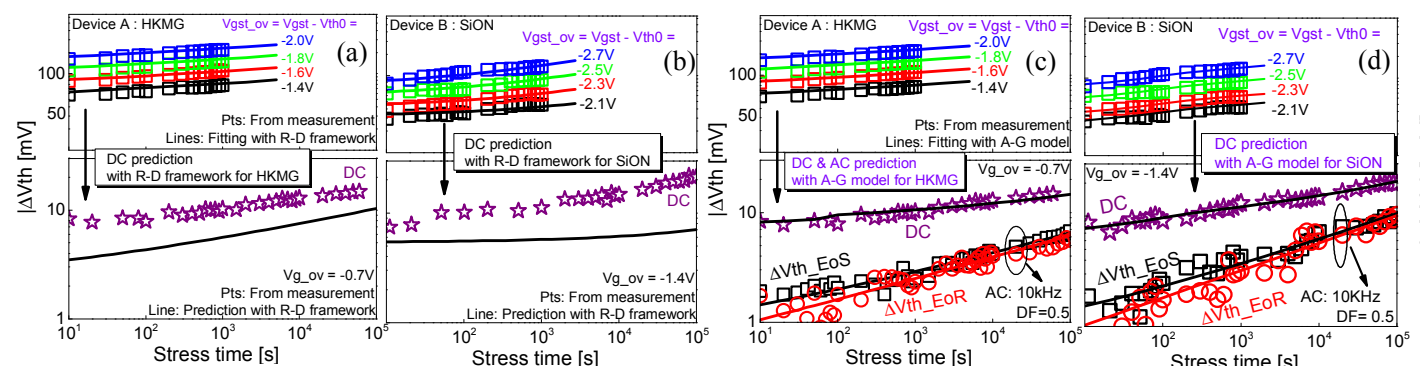

Fig.1 Demonstration of poor predictive capability of R-D based framework [2] (a\&b) and good capability of A-G model (c\&d) on devices with

HKMG and SiON gate stack. Symbols are test data and lines are calculated. The SAME four Vg-accelerated short DC stresses were used to extract model parameters for both R-D and A-G models (HKMG: a\&c; SiON: b\&d). The accelerated data can be fitted well by both models (the upper panels of a-d). The extracted R-D model, however, cannot predict the $\Delta \mathrm{Vth}$ under low use-Vg_ov, as shown by the difference between symbols and lines in the lower panels of $\mathbf{a} \& \mathbf{b})$. In contrast, the extracted A-G model predicts well not only for DC, but also for AC, NBTI under the SAME low use- $\mathrm{Vg} \_$ov (the lower panels of $c \& d$ ). The test data in the lower panels of a-d were not used for fitting.

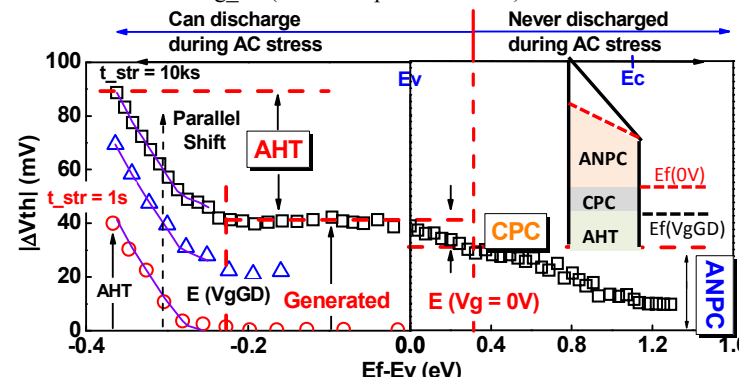

Fig.3 Defects separation based on their energy location. As-grown hole traps (AHT) are below E(VgGD) and the generated defects (GD) are above $\mathrm{E}(\mathrm{VgGD})$. GD is further separated into CPC between $\mathrm{E}(\mathrm{VgGD})$ and $\mathrm{E}(\mathrm{Vg}=0 \mathrm{~V})$ and $\mathrm{ANPC}$ above $\mathrm{E}(\mathrm{Vg}=0)$. For unipolar $\mathrm{AC}$, ANPC never discharges. The energy location of each defect is illustrated in the inset.
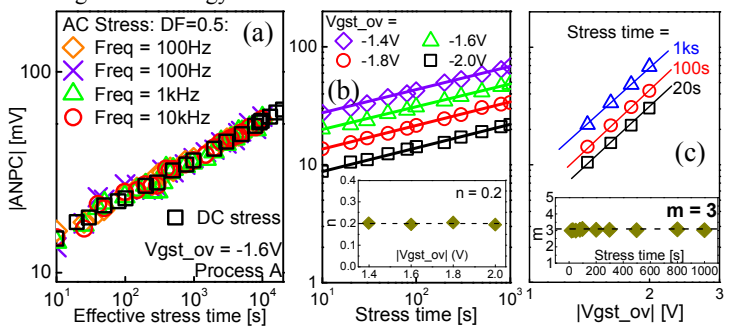

Fig 5 (a) A stress time $[\mathrm{s}]$.

ANPC DC agrees with ANPC_AC that is frequency-independent. (b) The generation kinetics of ANPC. The time exponent is independent of Vgst ov (inset). (c) ANPC for a given stress time follows a power law against Vgst_ov and the voltage exponent is independent of time (inset).
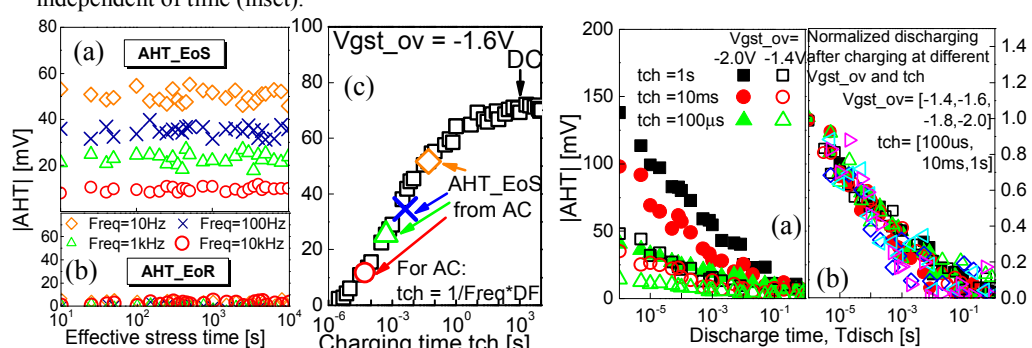

Fig.7 AHT at the end of AC stress (EoS) edge and end-of- Fig.8 (a) AHT discharge kinetics and (b) recovery (EoR) edge. (a) AHT EoS reduces for higher the normalization under different frequency. AHT_EoR is negligible for all frequencies. (b) Vgst_ov or charging time, tch. The For the same charging time, tch=period*DF (duty factor) kinetics is independent of Vgst_ov and

DC
MODE
(a) $00 \begin{array}{ll}\Delta V \operatorname{th}(V g s t o V) & \text { (c) } \\ \Delta V \operatorname{th}(V g G D)\end{array}$

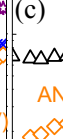

$\triangle \triangle \triangle \triangle \triangle M$

AC
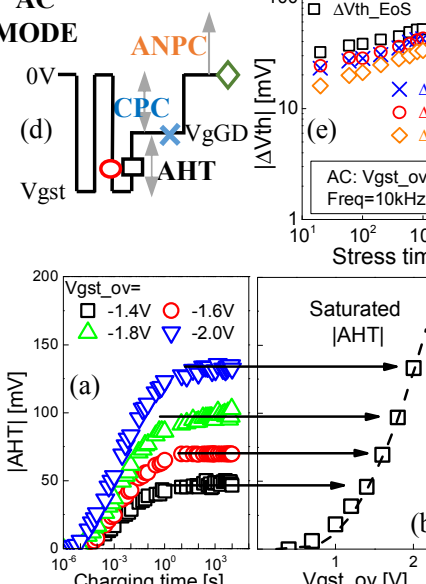

(b)

Vgst_ov [V]

$10^{-6}$

Fig.6 (a) AHT_DC kinetics under different Vgst_ov. It saturates in short time. (b) The experimentally measured saturation level under different Vgst ov. The dash line is a guard to the eye. (c) When AHT_DC is normalized against its saturation value, it follows the same charging kinetics.

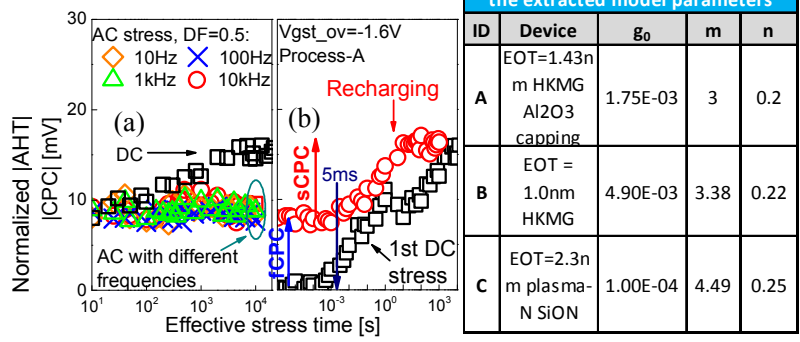

Fig.9 (a) A comparison of CPC extracted from DC and AC stress. Within 50s, $\mathrm{CPC} \mathrm{DC} \approx \mathrm{CPC} \mathrm{AC}$ regardless of frequency. For longer stress time, $\mathrm{CPC}$ saturates and CPC_DC $>$ CPC_AC. (b) A comparison of CPC DC under the $1^{\text {st }}$ stress and recharge. CPCs were fully discharged before recharge. Some CPCs were filled within $1 \mu \mathrm{s}$ ( $\mathrm{fCPC}$ ), whilst the rest only starts filling after $5 \mathrm{~ms}$ (sCPC). Total AC CPC in (a) agrees well with the fCPC in (b). for AC, AHT EoS agrees with AHT DC.

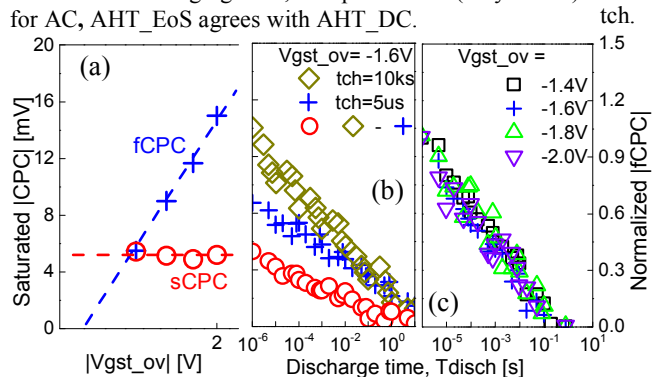

Fig.10 (a) The saturated $\mathrm{ACPC}$ and $\mathrm{SCPC}$ have different dependence on Vgst_ov. The extraction procedure is shown in Fig.9(b). (b) fCPC discharge kinetics ('+') is obtained by discharging after only $5 \mu$ s recharging on a stressed device. The SCPC discharge kinetics ('o') is obtained by subtracting fCPC from the total CPC (' $\vee$ '). (c) Normalized fCPC discharge kinetics is independent of Vgst ov.

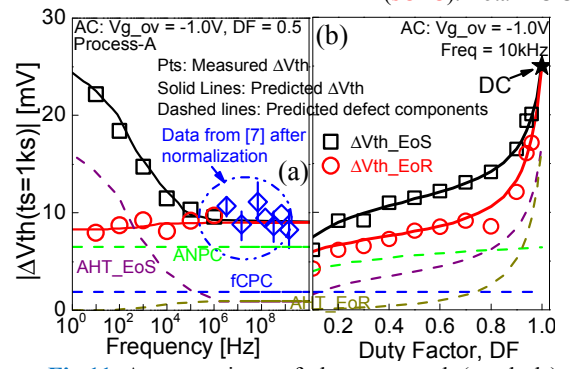

Fig.11 A comparison of the measured (symbols) and predicted (solid lines) Frequency (a) and Duty Factor (b) characteristics of AC-aging under low operating condition for process A. The detailed contribution of each type of defects are shown in dashed lines.

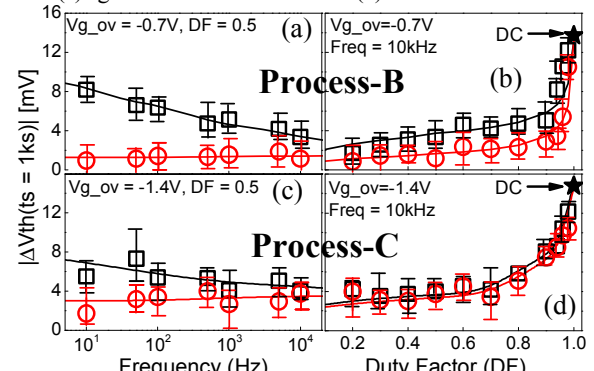

Frequency $(\mathrm{Hz})$ Duty Factor (DF)
Fig.12 A comparison of the measured (symbols) and predicted (lines) Frequency (a\&c) and Duty Factor (b-d) characteristics of AC-aging under lowest possible $\mathrm{Vg}$ for process $\mathrm{B} \& \mathrm{C}$. To improve the measurement accuracy, four samples are used for each stress condition and then averaged value is used. 